\title{
Perfil del usuario de centros deportivos privados, según género, edad y nivel de antigüedad
}

\section{User profile of private sports centers, by gender, age and seniority level}

\author{
Nuria Molina García ${ }^{1 *}$ J. Javier Mundina Gómez² y Ana Gómez Tafalla² \\ ${ }^{I}$ Departamento de Didáctica General y Didácticas Especificas. Universidad de Alicante, Alicante (España). \\ ${ }^{2}$ Departamento de Educación Física y Deportiva. Universidad de Valencia. Valencia (España).
}

\begin{abstract}
Resumen: En los últimos años ha surgido una extrema necesidad de perfilar a los usuarios de instalaciones deportivas debido al crecimiento en el sector tratando de ser cada vez más competitivos adecuando la oferta a la demanda (Nuviala et al., 2014). El objetivo del presente estudio fue definir el perfil del usuario, en función de la calidad, satisfacción, valor percibido e intenciones futuras, analizando si éstas varían según el género, la edad y el nivel de antigüedad, en 481 socios de tres centros deportivos privados. Para ello, se les administró un cuestionario y se realizó una prueba t- para muestras independientes y un estudio de contraste grupo a grupo con la prueba t- del test de Scheffé y Tamhane. Los resultados indicaron que las variables estudiadas fueron más positivas para el participante de género femenino, de más de 40 ańos y con una antigüedad en el centro menor a un año.

Palabras clave: Satisfacción, Calidad, Valor Percibido, Intenciones Futuras, Usuario, Centro Deportivo
\end{abstract}

Abstract: In recent years, there has been an extreme need to profile the users of sports facilities due to the growth in the sector, trying to be more and more competitive by adapting supply to demand (Nuviala et al., 2014). The aim of this study was to define the user's profile, based on quality, satisfaction, perceived value and future intentions, analyzing whether these vary according to gender, age and seniority level, in 481 members of three private sports centers. For this purpose, a questionnaire was administered and a t-test was carried out for independent samples and a group-to-group contrast study with the t-test of Scheffé and Tamhane's test. The results indicated that the variables studied were more positive for the female participant, who was over 40 years old and had been in the center for less than a year.

Keywords: Satisfaction, Quality, Perceived Value, Future Intentions, User, Sport Center

\section{Fundamentación teórica}

En Espańa, el sector privado de organizaciones de fitness es considerado como el líder del sector de las instalaciones de ocio (García, Lera-López y Suárez, 2011). Conocer cuáles son los motivos que llevan al usuario al abandono de un centro deportivo es fundamental para mejorar la gestión y poder trabajar para lograr la fidelidad de los usuarios matriculados en un centro deportivo (Martínez y Martínez 2009; Nuviala et al., 2012). En la misma línea, escuchar las experiencias de los usuarios es esencial en la implementación de cualquier tipo de servicio que pretenda mejorar la eficiencia y eficacia de su gestión y conseguir la fidelidad de los usuarios, por lo que resulta lógico que en los últimos años hayan aparecido numerosos estudios interesados en conocer las actitudes y comportamientos de los usuarios de centros deportivos (Martínez y Martínez, 2009; Nuviala et al., 2012b). Sin embargo, son escasos los estudios sobre las razones que llevan a los usuarios a causar baja en centros y organizaciones deportivas sin que ello suponga el abandono de la práctica de actividad física.

La importancia de detectar atributos que los clientes valo-

Dirección para correspondencia [Correspondence address]: Nuria Molina García. Departamento de Didáctica General y Didácticas Específicas. Universidad de Alicante. Educación Física y Deportes, C/ Aeroplano s/n, 03690, San Vicente del Raspeig, Alicante (España).

E-mail: n.molina@ua.es ran mejor en un servicio y los motivos por los cuáles toman esa elección, permiten a los gestores deportivos disponer de más información para una mejora en los planes de marketing y su desarrollo de actividades (Elasri et al., 2016). En los últimos años ha surgido una extrema necesidad de perfilar a los usuarios de instalaciones deportivas debido al crecimiento que ha experimentado el sector, teniendo como objetivo, ser utilizada como herramienta estratégica para responder a un mercado competitivo, así como para adecuar la oferta a la demanda (Nuviala et al., 2014). En el contexto deportivo, se estudia la segmentación de clientes que facilitará que los centros posean un valor añadido respecto a otros diferenciándose, pudiendo establecer políticas de precios, desarrollar servicios y crear campañas de publicidad adecuadas para el target que se persiga. Para Elasri et al. (2016), la segmentación de clientes consiste en identificar comportamientos similares entre personas de un determinado mercado, tratando de formar un grupo (o grupos) con características y comportamientos similares. Kotler y Armstrong (2004), consideran la segmentación como una herramienta clave para las estrategias de marketing actuales, de manera de que con esta división se pueda identificar mejor a los diversos grupos de clientes, ofreciéndoles servicios que satisfaga de forma única sus necesidades. Según Dixon, Backman, Backman y Norman (2012), crear grupos de clientes permite comprender mejor a los usuarios, 
y como consecuencia los gerentes pueden elaborar estrategias de comercialización y personalización de los servicios que ofrecen. Trabajos de García et al. (2016), informan de que el conocimiento de los clientes proporciona a las organizaciones deportivas información acerca del perfil determinado y características sociodemográficas de los usuarios de dichos perfiles, transmitiendo así información a los gerentes para que puedan satisfacer de forma más eficiente las necesidades de sus clientes, adaptándose a la diversidad de intereses y motivaciones existente (Funk, Filo, Beaton y Pritchard, 2009).

En el área de la gestión deportiva, existen estudios sobre la segmentación de clientes desde el punto de vista de la motivación (e.g., Nuviala et al., 2014), hasta otros que tratan sobre el punto de vista de la satisfacción a partir de la calidad y el valor percibido en eventos deportivos (e.g. Calabuig, Burillo, Crespo, Mundina y Gallardo, 2010). Algunos trabajos como el de Rial, Alonso, Rial, Picón y Varela (2009), se han centrado en analizar los diferentes perfiles de usuarios de centros deportivos privados, concluyendo que los clientes no son homogéneos y que, según las variables, se necesitaría un trato diferente para cada uno de ellos. Aun así, existen escasos estudios que trabajen la segmentación de grupos basándose en variables como la satisfacción del cliente (Elasri-Ejjaberi et al., 2016). Autores como Kotler y Armstrong (2004), proponen una única manera de segmentar el mercado centrándose las investigaciones del sector deportivo en aplicaciones de diferentes técnicas tanto de forma individual como combinada tipo geográfica, demográfica o comportamental. Por otro lado, estudios de Luna- Arocas y Li-Ping (2005), analizan los perfiles del cliente en base a las motivaciones y la satisfacción en centros de fitness en España. Siguiendo la misma línea, García, Fernández y Bernal (2014), tienen en cuenta las variables sociodemográficas, y concluyen que las mujeres tienen mejores intenciones futuras.

Como objetivo, esta investigación pretende definir el perfil del usuario en función de la valoración de las variables de calidad, satisfacción, valor percibido e intenciones futuras y analizar si varían en función de los grupos definidos de edad, género, y nivel de antigüedad como socio, en los usuarios de tres centros deportivos de gestión privada.

\section{Método}

Para llevar a cabo este trabajo se utilizó un cuestionario de 98 ítems cumplimentado por 481 socios pertenecientes a 3 centros deportivos privados de la provincia de Alicante. Esta muestra, estuvo representada en un $52,6 \%$ por hombres $(\mathrm{N}=253)$ y un $47,4 \%$ mujeres $(\mathrm{N}=228)$. Para el procedimiento, se administraron cuestionarios en papel impreso distribuidos por personal cualificado del centro y debidamente informado, y en modo online a través de la plataforma Lime-Survey, mediante la cual los usuarios accedían a través de un enlace y cumplimentaban el cuestionario de forma anónima. Los instrumentos utilizados para medir las variables de calidad y satisfacción general fueron la escala de Hightower, Brady y Baker (2002). Para medir la calidad se utilizaron 3 de los 5 ítems de la escala compuesta, y para medir la satisfacción 3 ítems siendo ambas escalas compuestas por preguntas con alternativa de respuesta tipo Likert de 5 puntos.

Para continuar con el análisis, se midió el valor percibido con la escala PERVAL de Sweeney y Soutar (2001), siendo ésta la escala de valor más rigurosa y robusta por las tres dimensiones que analiza a través de 7 ítems y una opción de respuesta de 1 a 5 tipo Likert. Estas dimensiones son; dimensión funcional (económica y de calidad), dimensión social y dimensión emocional.

Finalmente, la escala que se ocupó de medir intenciones futuras a través de 4 ítems fue la de Zeithaml, Berry y Parasuraman (1996), donde se pregunta por la lealtad en un ítem y por WOM (Word of mouth) en los tres restantes para evaluar la intención del usuario de volver y de recomendar el servicio a otros.

Estas variables fueron medidas, evaluadas, y posteriormente se compararon sus resultados y diferencias en función de tres agrupaciones: un primer grupo entre género femenino y masculino, un segundo grupo entre la edad de los usuarios segmentando la muestra en tres sub-grupos: uno de menores de 25 años, otro grupo de entre 26 y 40 años y uno de usuarios de más de 40 años de edad. Y finalmente, un tercer grupo según la antigüedad como socio en el centro del usuario, siendo este novel si tenía una permanencia de entre 1 y 12 meses, y veterano si su permanencia era mayor a 13 meses como socio en el centro deportivo.

Para el tratamiento de los datos se utilizó el paquete estadístico SPSS 24.0 con licencia de la Universidad de Alicante y se realizaron diferentes técnicas de análisis de datos que pasamos a detallar a continuación:

Se llevó a cabo un análisis descriptivo de las variables en valores absolutos de frecuencias y porcentajes de edad, género y antigüedad como socio en el centro. Posteriormente se realizó un análisis factorial exploratorio de las escalas de calidad general, satisfacción general, valor percibido e intenciones futuras, y se realizaron cálculos de parámetros descriptivos de media, desviación típica y varianza. Para el análisis de fiabilidad se empleó el cálculo estadístico Alfa de Cronbach como modelo de fiabilidad.

Para comparar los grupos se realizó la prueba $\mathrm{T}$ para muestras independientes y a posteriori un contraste grupo a grupo con el test de Scheffé y Tamhane.

Las distintas escalas utilizadas fueron sometidas a análisis de fiabilidad y validez para reportar información sobre sus propiedades psicométricas presentando todas ellas buenos índices de fiabilidad y consistencia interna con un Alfa de Cronbach superior , 80 . 


\section{Resultados}

A continuación, se muestran los resultados obtenidos comenzando por los descriptivos obtenidos de la muestra, las escalas utilizadas y posteriormente, se presentan las diferencias de medias existentes entre los grupos observados; el género, la antigüedad como socio en el centro y los tramos de edad.
En la tabla 1, se puede observar como todas las escalas utilizadas muestran buenos índices de fiabilidad como consistencia interna, pues el alfa de Cronbach oscila entre ,88 ,95. Además, se muestra el número de ítems de cada escala y se detalla si se utilizó el total de ítems de cada escala o se eliminó alguno para el análisis estadístico.

Tabla 1. Análisis de fiabilidad de las escalas utilizadas.

\begin{tabular}{cccc}
\hline Nombre de las Escalas & Alfa de Cronbach & No de ítems & Ítems \\
\hline Calidad general &, 95 & 3 & Todos \\
Satisfacción general &, 92 & 3 & Todos \\
Valor emocional &, 88 & 2 & Se elimina el ítem 49 \\
Valor social &, 89 & 2 & Todos \\
Valor precio &, 88 & 2 & Todos \\
Intenciones futuras &, 93 & 4 & Todos \\
\hline
\end{tabular}

En referencia a la tabla 1, se ha eliminado un ítem de la escala de valor percibido en la dimensión de valor emocional ya que al eliminar el elemento mejoraba la fiabilidad de la misma.

En cuanto a la valoración de la calidad general, en la tabla 2, se observan puntuaciones positivas en los 3 ítems utilizados de los 5 que componen la escala de Hightower et al. (2002). Con una alternativa de respuesta de 1 a 5 , los valores medios para una muestra de 481 participantes fueron de mayores a 4 en todos ellos, siendo el primer ítem el mejor valorado con 3,94 (D.T. ,86) y el conjunto con una valoración media de 3,89 (D.T. ,82).

Tabla 2. Descriptivos y valoración de medias de la calidad general.

\begin{tabular}{lcc}
\hline & Media & D.T. \\
\hline En general, he recibido un servicio de alta calidad en la instalación & 3,94 &, 86 \\
Generalmente, el servicio ofrecido en la instalación es excelente. & 3,88 &, 88 \\
En general, el servicio ofrecido en la instalación es sobresaliente. & 3,85 &, 92 \\
\multicolumn{1}{c}{ Media de calidad general } & 3,89 &, 82 \\
\hline
\end{tabular}

En cuanto a la valoración de la satisfacción general, se puede observar en la tabla 3, cómo las puntuaciones de la muestra de los participantes son altas en todos los ítems y mayores a 4 con una valoración media de la satisfacción general de 4,11 (D.T. ,87). En la variable, que se mide a través de 3 ítems con una opción de respuesta de 1 a 5, el ítem mejor valorado ha sido el tercero donde se afirma que: verdaderamente he disfrutado asistiendo a la instalación, valorado con una puntuación media de 4,11 (D.T. ,87).

Tabla 3. Descriptivos y valoración de medias de la satisfacción general.

\begin{tabular}{lcc}
\hline & Media & D.T. \\
\hline Estoy contento/a con las experiencias que he tenido en la instalación. & 4,08 &, 88 \\
Estoy satisfecho/a con mis experiencias en la práctica deportiva. & 4,12 &, 85 \\
Verdaderamente he disfrutado asistiendo a la instalación. & 4,11 &, 87 \\
Media de satisfacción general & 4,11 &, 78 \\
\hline
\end{tabular}

Se debe de destacar que la satisfacción general fue la variable mejor valorada en su conjunto con 4,11 (D.T. ,78) de entre todas las variables generales de calidad y satisfacción general, valor emocional, valor precio, valor social e intenciones futuras.
En cuanto a la valoración del valor percibido, y sus dimensiones de valor emocional, valor precio y valor social, en la tabla 4 se perciben las valoraciones medias de los participantes a través de la escala PERVAL de Sweeney y Soutar (2001). Esta escala la componen 7 ítems de los cuáles se utilizaron 6 para 
el cuestionario ya que se eliminó uno de ellos de la dimensión valor emocional para mejorar la fiabilidad de la escala. Si observamos los resultados obtenidos en la tabla 4, podemos apreciar que el valor emocional fue la dimensión con las puntuaciones más altas con valores medios de 4,04 (D.T. ,86) preguntando por las emociones que produce la práctica deportiva en la instalación. Destacar, que la dimensión de valor social fue la variable que obtuvo puntuaciones más bajas de 3,78 (D.T. ,96) sobre 5, de entre todas las variables estudiadas.

Tabla 4. Descriptivos y valoración de medias del valor percibido.

\begin{tabular}{|c|c|c|}
\hline & Media & D.T. \\
\hline Hacer deporte aquí hace que tenga ganas de volver. & 4,03 & ,92 \\
\hline Venir a la instalación me aporta cierto placer. & 4,05 & ,89 \\
\hline Media de valor emocional & 4,04 & ,86 \\
\hline La instalación tiene un precio razonable. & 3,84 & 1,03 \\
\hline La instalación ofrece un buen servicio por lo que he pagado. & 3,87 & 1,00 \\
\hline Media de valor precio & 3,90 & 91 \\
\hline El hacer deporte aquí mejora la forma en que soy percibido/a. & 3,74 & 1,03 \\
\hline Venir a esta instalación causa una buena impresión en los demás. & 3,71 & 1,07 \\
\hline Media de valor social & 3,78 & ,96 \\
\hline
\end{tabular}

Finalmente, para la valoración de las intenciones futuras, como se puede comprobar en la tabla 5, la escala fue puntuada con niveles altos y de forma positiva con valores medios de 4,10 (D.T. ,83), siendo los ítems mejor valorados el de lealtad que pregunta si estoy dispuesto/a a continuar asistiendo a la instalación el año/ curso que viene con una puntuación de 4,10 (D.T. ,97), y el de recomendar el servicio con valores de 4,10 (D.T. ,93) sobre 5.

Tabla 5. Descriptivos y valoración de medias de las intenciones futuras.

\begin{tabular}{lcc}
\hline & Media & D.T. \\
\hline Estoy dispuesto/a a continuar asistiendo a la instalación el año/curso que viene & 4,10 & 4,04 \\
Recomendaré venir a esta instalación a otras personas & 4,06 &, 98 \\
Animaré a otras personas para que se apunten a esta instalación & $4,10 \quad, 96$ \\
Normalmente hablo bien de los servicios que ofrece esta instalación & Media de intenciones futuras & $4,10 \quad 83$ \\
\hline
\end{tabular}

Destacar que las intenciones futuras es la segunda variable mejor valorada 4,10 (D.T. ,83), después de la satisfacción general de entre todas las variables generales de calidad y satisfacción general, valor emocional, valor precio, valor social e intenciones futuras.

En cuanto al análisis de los resultados de las variables según los grupos establecidos de género, antigüedad como socio en el centro y tramos de edad, los resultados obtenidos que se muestran en la tabla 6 , indican que los participantes de género femenino valoran con puntuaciones más altas que los hombres las variables de calidad general, satisfacción, valor emocional, valor precio, valor social e intenciones futuras. Destacar que, según el género, las variables de satisfacción general y valor emocional presentan diferencias estadísticamente significativas $(\mathrm{p}<, 05)$. Seguidamente, en la misma tabla, podemos percibir que, en casi todas las variables estudiadas, las valoraciones de los participantes noveles (1 a 12 meses) son más altas y positivas que las de los clientes veteranos (más de 13 meses en el centro), a excepción de la variable de valor precio en la cual las puntuaciones son muy similares en ambos grupos de antigüedad y mínimamente mejor valorada por el grupo de veteranos. Cabe seńalar que, en este grupo de antigüedad, las variables de calidad general, satisfacción general y valor emocional presentan diferencias estadísticamente significativas $(\mathrm{p}<, 05)$. Del mismo modo en la tabla 6 , se observa que existe una relación positiva a valorar mejor todas las variables de la tabla a mayor edad, puesto que el grupo de más de 40 años es el que otorga valoraciones más positivas a todas las variables, siendo en este caso, las variables de valor emocional e intenciones futuras las que cuentan con diferencias estadísticamente significativas $(\mathrm{p}<, 05)$ en los tres sub grupos de edad y valor precio la que cuenta con diferencias significativas en los sub grupos de participante de menos de 25 años y de más de 40 años de edad. 
Tabla 6. Descriptivos de las valoraciones según género, antigüedad en el centro y grupos de edad.

\begin{tabular}{|c|c|c|c|c|c|c|c|c|c|c|c|c|}
\hline & Sexo & $\mathrm{N}$ & $\mathrm{X}$ & DT & Antigüedad & $\mathrm{N}$ & $\mathrm{X}$ & DT & Edad & $\mathrm{N}$ & $\mathrm{X}$ & DT \\
\hline Calidad General & $\begin{array}{l}\mathrm{H} \\
\mathrm{M}\end{array}$ & $\begin{array}{l}253 \\
228\end{array}$ & $\begin{array}{l}3,83 \\
3,95\end{array}$ & $\begin{array}{l}, 87 \\
, 75\end{array}$ & $\begin{array}{c}\text { Noveles } \\
\text { Veteranos }\end{array}$ & $\begin{array}{l}243 \\
238\end{array}$ & $\begin{array}{c}4,05 \\
3,72^{* * *}\end{array}$ & $\begin{array}{l}, 72 \\
, 88\end{array}$ & $\begin{array}{c}<25 \\
26-40 \\
>40\end{array}$ & $\begin{array}{l}109 \\
233 \\
139\end{array}$ & $\begin{array}{l}3,84 \\
3,83 \\
4,01\end{array}$ & $\begin{array}{l}, 97 \\
, 75 \\
, 79\end{array}$ \\
\hline Satisfacción General & $\begin{array}{l}\mathrm{H} \\
\mathrm{M}\end{array}$ & $\begin{array}{l}253 \\
228\end{array}$ & $\begin{array}{l}4,04 \\
4,18^{*}\end{array}$ & $\begin{array}{l}, 81 \\
, 76\end{array}$ & $\begin{array}{c}\text { Noveles } \\
\text { Veteranos }\end{array}$ & $\begin{array}{l}243 \\
238\end{array}$ & $\begin{array}{c}4,22 \\
3,99^{* * *}\end{array}$ & $\begin{array}{l}, 72 \\
, 83\end{array}$ & $\begin{array}{c}<25 \\
26-40 \\
>40\end{array}$ & $\begin{array}{l}109 \\
233 \\
139\end{array}$ & $\begin{array}{l}4,05 \\
4,05 \\
4,23\end{array}$ & $\begin{array}{l}, 89 \\
, 73 \\
, 77\end{array}$ \\
\hline Valor Emocional & $\begin{array}{l}\mathrm{H} \\
\mathrm{M}\end{array}$ & $\begin{array}{l}253 \\
228\end{array}$ & $\begin{array}{l}3,97 \\
4,11^{*}\end{array}$ & $\begin{array}{l}, 86 \\
, 86\end{array}$ & $\begin{array}{c}\text { Noveles } \\
\text { Veteranos }\end{array}$ & $\begin{array}{l}243 \\
238\end{array}$ & $\begin{array}{c}4,13 \\
3,91^{* *}\end{array}$ & $\begin{array}{l}, 87 \\
, 89\end{array}$ & $\begin{array}{c}<25^{*} \\
26-40^{*} \\
>40^{* *}\end{array}$ & $\begin{array}{l}109 \\
233 \\
139\end{array}$ & $\begin{array}{l}3,95 \\
3,94 \\
4,24\end{array}$ & $\begin{array}{l}, 90 \\
, 79 \\
, 82\end{array}$ \\
\hline Valor Precio & $\begin{array}{l}\mathrm{H} \\
\mathrm{M}\end{array}$ & $\begin{array}{l}253 \\
228\end{array}$ & $\begin{array}{l}3,87 \\
3,94\end{array}$ & $\begin{array}{l}, 93 \\
, 86\end{array}$ & $\begin{array}{c}\text { Noveles } \\
\text { Veteranos }\end{array}$ & $\begin{array}{l}243 \\
238\end{array}$ & $\begin{array}{l}3,86 \\
3,94\end{array}$ & $\begin{array}{l}, 84 \\
, 97\end{array}$ & $\begin{array}{c}<25^{*} \\
26-40 \\
>40^{*}\end{array}$ & $\begin{array}{l}109 \\
233 \\
139\end{array}$ & $\begin{array}{l}3,75 \\
3,88 \\
4,06\end{array}$ & $\begin{array}{l}, 92 \\
, 85 \\
, 97\end{array}$ \\
\hline Valor Social & $\begin{array}{l}\mathrm{H} \\
\mathrm{M}\end{array}$ & $\begin{array}{l}253 \\
228\end{array}$ & $\begin{array}{l}3,77 \\
3,80\end{array}$ & $\begin{array}{l}, 95 \\
, 97\end{array}$ & $\begin{array}{c}\text { Noveles } \\
\text { Veteranos }\end{array}$ & $\begin{array}{l}243 \\
238\end{array}$ & $\begin{array}{l}3,79 \\
3,77\end{array}$ & $\begin{array}{l}, 94 \\
, 99\end{array}$ & $\begin{array}{c}<25 \\
26-40 \\
>40\end{array}$ & $\begin{array}{l}109 \\
233 \\
139\end{array}$ & $\begin{array}{l}3,68 \\
3,73 \\
3,96\end{array}$ & $\begin{array}{l}1,05 \\
, 88 \\
, 99\end{array}$ \\
\hline Intenciones Futuras & $\begin{array}{l}\mathrm{H} \\
\mathrm{M}\end{array}$ & $\begin{array}{l}253 \\
228\end{array}$ & $\begin{array}{l}4,02 \\
4,19^{*}\end{array}$ & $\begin{array}{l}, 88 \\
, 76\end{array}$ & $\begin{array}{c}\text { Noveles } \\
\text { Veteranos }\end{array}$ & $\begin{array}{l}243 \\
238\end{array}$ & $\begin{array}{l}4,16 \\
4,03\end{array}$ & $\begin{array}{l}, 79 \\
, 87\end{array}$ & $\begin{array}{c}<25^{*} \\
26-40^{*} \\
>40^{* * *} \\
\end{array}$ & $\begin{array}{l}109 \\
233 \\
139 \\
\end{array}$ & $\begin{array}{l}3,96 \\
4,00 \\
4,37 \\
\end{array}$ & $\begin{array}{l}, 92 \\
, 81 \\
, 71 \\
\end{array}$ \\
\hline
\end{tabular}

Nota: ${ }^{*} \mathrm{p}<, 05,{ }^{* *} \mathrm{p}<, 01 ;{ }^{* * *} \mathrm{p}<, 001$.

\section{Discusión y conclusiones}

Este trabajo persigue como objetivo definir el perfil del usuario de centros deportivos privados en función a las variables de calidad, satisfacción general, valor percibido e intenciones futuras en función del género, la antigüedad como socio en el centro y tramos de edad. Los resultados obtenidos reflejan la valoración de cada variable en función de los grupos elegidos o segmentados detectando lo siguiente:

Las variables de calidad, satisfacción general, valor percibido e intenciones futuras son valoradas de forma más alta y positiva por el participante de género femenino que por el masculino. Confirmando los resultados de trabajos encontrados de García et al. (2014), y Bernal (2014), en los cuales concluyen que las mujeres tienen mejores intenciones futuras, pero no presentan diferencias estadísticamente significativas en cuanto al a satisfacción del usuario. En estudios de Calabuig et al. (2010), se obtienen resultados más positivos por parte de las mujeres, pero se discrepa sobre su relación ya que parece que existen argumentos que cuestionan que estas diferencias pueden deberse al deporte analizado o al tipo de evento.

Respecto a la comparación de las medias de las variables respecto a los tramos de edad de $<25$, entre 26 y 40 y $>$ de 40 años de edad, los resultados del presente estudio afirman que a mayor edad del usuario las valoraciones son más altas y positivas en todas las variables. Estos resultados son acordes a los obtenidos por Triadó, Aparicio y Rimbau (1999), los cuales encontraron diferencias estadísticamente significativas entre los grupos de mayor edad y los de menor edad respecto a su satisfacción con unos servicios deportivos municipales. Contrariamente estudios de Bernal (2014), difieren afirmando que el rango de edad con mejor valoración para la satisfacción del usuario es menor a 15 años, siendo datos similares a trabajos de Calabuig et al. (2008), en los cuales se afirma que, a mayor edad, menor es su satisfacción, por lo que se contradice con los datos obtenidos en el presente estudio.

Como último grupo, se evaluaron las variables según la antigüedad como socio en el centro del usuario y se concluyó que los participantes o usuarios con una antigüedad como socio en el centro de entre 1 y 12 meses, es decir noveles, valoraban de forma más positiva todas las variables estudiadas presentando diferencias estadísticamente significativas en las variables de calidad general, satisfacción general y valor emocional. Son muy escasos los estudios que relacionan a las variables con este grupo, pero sí que se encuentran trabajos que tratan de crear programas que alarguen la vida del usuario en los centros deportivos de forma continua y eviten así las bajas de los mismos con modelos predictivos de bajas de centros deportivos como lo son, trabajos de Clavel (2017), o Teva-Villén et al. (2014), los cuales estudian la segmentación de los clientes con la intención de aumentar la lealtad, prevenir bajas y aumentar la satisfaccion e intenciones futuras de los clientes para que aumente la antiguedad de éstos en los centros deportivos.

Como conclusión, los datos obtenidos en este trabajo nos indican que el usuario de centros deportivos privados con mejor valoración en las variables de calidad, satisfacción, valor percibido e intenciones futuras sería un participante de género femenino, con una antigüedad novel como usuario/a en 
el centro de 1 a 12 meses y de una edad superior a los 40 años.

Es de destacar que, todas las dimensiones estudiadas presentaron diferencias estadísticamente significativas en alguna de las agrupaciones, destacando el valor percibido con diferencias significativas en sus tres agrupaciones.

\section{Limitaciones y futuras lineas de investigación}

Como futuras líneas de investigación se propone contrastar estos datos en función del tipo de servicio en cuanto a su gestión para evaluar si existen diferencias entre el tipo de perfil de usuario entre centros deportivos públicos y privados. Además, sería interesante completar la información y resultados obtenidos en cuanto a satisfacción e intenciones futuras con aspectos conductuales del usuario posteriores a éstos como pueden ser la confirmación de permanencia en cuanto al cliente satisfecho o la medición de bajas o re-altas en el mismo centro deportivo, tras un periodo de baja de aquellos clientes o usuarios con una satisfacción y valoración de las variables más baja.

\section{Referencias}

1. Bernal García, A. (2014). Fidelización de clientes en organizaciones deportivas: calidad, valor percibido y satisfacción como factores determinantes. Tesis doctoral inédita, Universidad de Sevilla, Sevilla. Recuperado de https://idus.us.es/xmlui/handle/11441/53492.

2. Calabuig, F., Quintanilla, I. y Mundina, J. J. (2008). La calidad percibida de los servicios deportivos: Diferencias según instalación, género, edad y tipo de usuario en servicios náuticos. Revista Internacional de Ciencias del Deporte, 4(10), 25-43

3. Calabuig, F., Burillo, P., Crespo, J., Mundina. J., y Gallardo, L. (2010). Satisfacción, calidad y valor percibido en espectadores de atletismo. International Journal of Medicine and Science of Physical Activity and Sport, 10(40), 577-593.

4. Calabuig, F., Crespo, J., Núñez, J., Valantine, I., \& Staskeviciute-Butiene, I. (2016). Role of Perceived Value and Emotions in the Satisfaction and Future Intentions of Spectators in Sporting Events. Inzerine Ekonomika-Engineering Economics, 27(2), 221-229.

5. Clavel San Emeterio, I. (2017). Creación de un modelo predictivo de bajas en centros deportivos.

6. Elasri-Ejjaberi, A., Triadó-Ivern, X., y Aparicio-Chueca, P. (2016). Los usuarios de los centros deportivos públicos: una aproximación de segmentación sobre hábitos deportivos y satisfacción. Revista de Psicología del Deporte, 25(1), 15-18.

7. Dixon, A., Backman, S., Backman, K. y Norman, W. (2012). Expenditure-based segmentation of sport tourists. Journal of Sport \& Tourism, 17(1), 5-21

8. Funk, D. C., Filo, K., Beaton, A. A. y Pritchard, M. (2009). Measuring the motives of sport event attendance: Bridging the academic-practitioner divide to understanding behaviour. Sports Marketing Quarterly, 18, 126-138.

9. García, J., Gálvez, P., Bernal, A. y Vélez, L. (2016). El gasto económico en centros de fitness low-cost: Diferencias según fidelidad y características del cliente. Sport TK: Revista Euroamericana de Ciencias del Deporte, 5,137-144

10. García , J., Lera-López, F. y Suárez, M. (2011). Estimation of a structural model of the determinants of the time spent on physical activity and sport: Evidence for Spain. Journal of Sports Economics, 12(5), 515-537.

11. García, J., Fernández, J. y Bernal, A. (2014). La percepción de calidad y fidelidad en clientes de centros de fitness low-cost. Suma Psicológica, 21(2), 123-130.

12. Hightower, r., Brady, M.K. y Baker, T.L. (2002). Investigating the role of the physical environment in hedonic service consumption: An exploratory study of sporty events. Journal of Business Reserach, 55, 697-707.

13. Kotler, P., Armstrong, G., Cámara Ibañez, D. y Cruz Roche, I. (2004). Marketing (10a ed.). Madrid: Pearson Educación.

14. Martinez, Jose A.; Martínez, Leticia. (2009). A customer management model in sports services; a system dynamics approach. Revista Internacional de Medicina y Ciencias de la Actividad Física y del Deporte, 9(36), 431-453.

15. Nuviala, A., Grao-Cruces, A., Pérez-Turpin, J. A., \& Nuviala, R. (2012a). Perceived service quality, perceived value and satisfaction in groups of users of sports organizations in Spain. Kinesiology, 44(1), 94103.

16. Nuviala, A, Pérez-Ordás, R., Boceta Osuna, M., Grao-Cruces, A., Nuviala, R., \& González Jurado, J. A. (2012b). Calidad, satisfacción y valor percibido de los usuarios de un servicio deportivo público. Movimento, 18(4) 11-32.

17. Nuviala, R., Teva-Villén, M. R., Pérez-Ordás, R., Grao-Cruces, A., Tamayo Fajardo, J. A., y Nuviala, A. (2014). Segmentción de usuarios de servicios deportivos. Retos. Nuevas Tendencias en Educación Física, Deporte y Recreación, 25, 90-94.

18. Rial, A., Alonso, D., Rial, J., Picón, E. y Varela, J. (2009). Un intento de segmentación integral de los usuarios de centros deportivos. Apunts, Educación Física y Deportes, 95, 82-91.

19. Sweeney, J., \& Soutar, G. (2001). Consumer perceived value: the development of a multiple item scale. Journal of Retailing, 77, 203-220.

20. Teva-Villén, M., Pérez-Ordás, R., Grao-Cruces, A., Tamayo- Fajardo J., Nuviala, R., y Nuviala, A. (2014). Abandono de ususarios de un centro deportivo español: segmentación. Movimiento, 20(2), 619-635.

21. Triadó, X., Aparicio, P. y Rimbau, E. (1999). Identification of factors of customer satisfaction in municipal sport centres in Barcelona. Some suggestions for satisfaction improvement. The Cyber-Journal of Sport Marketing, 3(4).

22. Zeithaml, V. A., Berry, L.L. y Parasuraman, A. (1996). The behavioural consequences of service quality. Journal of Marketing, 60(2), 31-46. 